\title{
Genotypic Determination of Carbapenamase Gene Production in Clinical Isolates of Klebsiella Pneumoniae in the University of Port-Harcourt Teaching Hospital
}

\author{
Igunma Agbons Jeremiah ${ }^{1}$, Akujobi Comfort Nne ${ }^{2}$, Oboro Ibinabo Laura ${ }^{1, *}$ \\ ${ }^{1}$ Department of Medical Microbiology and Parasitology, University of Port Harcourt Teaching Hospital, Port Harcourt, Nigeria \\ ${ }^{2}$ Department of Medical Microbiology and Parasitology, Nnamdi Azikiwe University Teaching Hospital, Nnewi, Nigeria
}

Email address:

ibinabo.oboro@gmail.com(O. I. Laura)

${ }^{*}$ Corresponding author

\section{To cite this article:}

Igunma Agbons Jeremiah, Akujobi Comfort Nne, Oboro Ibinabo Laura. Genotypic Determination of Carbapenamase Gene Production in Clinical Isolates of Klebsiella Pneumoniae in the University of Port-Harcourt Teaching Hospital. American Journal of Laboratory Medicine. Vol. 5, No. 3, 2020, pp. 70-75. doi: 10.11648/j.ajlm.20200503.12

Received: June 27, 2020; Accepted: July 13, 2020; Published: July 30, 2020

\begin{abstract}
Carbapenem resistance among Enterobacteriaceae, especially Klebsiella pneumoniae and Escherichia coli, has remained a challenge in infectious disease management. Although several resistance mechanisms have been documented that neutralize the efficacy of carbapenems, the expression of carbapenemases is the most prominent. Carbapenemase producing Klebsiella pneumoniae has been identified as a major public health threat because of the potential for rapid plasmid mediated spread of the resistance genes among bacterial species and resulting limited therapeutic options available. These pathogens could express resistance to almost all available antibiotics and are associated with high morbidity and mortality. This crosssectional study was carried out in the University of Port Harcourt Teaching Hospital, Nigeria. A total of 225 clinical isolates of Klebsiella pneumoniae from wounds, urine, blood, sputum and cerebrospinal fluid were recruited. All isolates were screened for Carbapenem resistance using Ertapenem (10 $\mu$ g; Oxoid, England) according to CLSI guidelines. All non-susceptible isolates were then tested for phenotypic carbapenemase production using the Modified Hodge test (MHT). The MHT positive isolates were thereafter, subjected to Polymerase Chain Reaction to detect the presence of blaKPC resistance gene. The study showed that 8.4\% (19/225) of Klebsiella pneumoniae isolates were not susceptible to Ertapenem. Out of these, 6.2\% (14/225) expressed carbapenemase production by the modified Hodge test. However, on molecular analysis, only six (6) of these isolates possessed the blaKPC gene giving a 2.7\% genotypic prevalence of Klebsiella pneumoniae carbapenemase among the isolates. Critically ill patients are more likely to develop infections by blaKPC-producing Klebsiella pneumoniae. It is therefore pertinent that the approach to prevention and control of infections by multi-drug resistant Klebsiella pneumoniae isolates must be multi-pronged to effectively counteract the various mechanisms that may be responsible. From control of prescribing and dispensing patterns, rational use of antimicrobials, institution of antimicrobial stewardship programs, optimization of infection prevention and control measures to effective surveillance; a well-coordinated approach is necessary to reduce the prevalence and spread of this pathogens since the blaKPC resistance gene is plasmid mediated and associated with high rates of both inter and intra-species transfer among bacteria.
\end{abstract}

Keywords: KPC, Klebsiella pneumoniae, Multidrug Resistance, Carbapenemase

\section{Introduction}

The world's greatest threats in current management of bacterial infections is the emergence of multi-drug resistant (MDR) bacteria which has resulted in limited treatment options even to the seemingly uncomplicated and easy to treat infections $[1,2]$. This phenomenon is more pronounced among infections caused by Gram negative organisms especially of the Enterobacteriaceae family, and it is increasingly being reported both in hospital and community settings worldwide [3]. Klebsiella species colonize the 
human gastrointestinal tract, upper respiratory tract, bladder and skin, and could multiply and survive in moist areas in the healthcare environment [4]. Most strains of this organism accumulate plasmids that carry virulence and resistance genes which facilitate the wide transfer of these genes within (intra) and between (inter) species conferring them with the ability to resist the major antibiotics used for their treatment such as cephalosporins, carbapenems, penicillins, aminoglycosides or fluoroquinolones [5].

Carbapenem antibiotics are known for their wide range of activity against gram negative organisms; particularly those producing extended-spectrum $\beta$-lactamase enzymes, as well as a wide range of Gram-positive bacteria. This quality earned it the tag "the antibiotics of last resort" [6]. However, this attribute had come under great threat following the emergence and increasing spread of carbapenemase producing bacteria [6]. Carbapenemases have emerged in various parts of the world, including Europe, the United States of America (USA), and eastern countries [7]. Carbapenem-resistant Klebsiella pneumoniae are strains of Klebsiella pneumoniae expressing the carbapenemase enzymes which confer them with resistance to all or nearly all available antibiotics, including carbapenems [8]. The Ambler class-A carbapenemase, KPC is the most commonly reported [7]. Klebsiella pneumoniae carbapenemase (KPC)-producing clinical isolates have been associated with several hospital outbreaks in the USA, Asia, Europe, South Korea and Central and South America [9]. In Africa, various studies have shown increasing prevalence of KPC producing Klebsiella pneumoniae in South Africa and Egypt [10]. In Nigeria, a prevalence of $16.7 \%$ was found in a study done in Jos, Plateau state [11].

The spread of antimicrobial resistance is not limited by boundaries neither does it discriminate between the specimens from which the organisms were isolated especially in the setting of poor infection control practices [12]. The blaKPC gene is a plasmid encoded gene that is easily transferred to susceptible bacteria at a high frequency and limited by only adequate and coordinated infection prevention and control practices [13].

Several MDR Klebsiella pneumoniae isolates have been identified in the Medical Microbiology laboratory of the University of Port-Harcourt teaching hospital (UPTH), notably from samples received from the neonatal and adult intensive care units. Also, risk factors known to predispose to spread of KPC, which includes (but not limited to) irrational use of antibiotics both in the hospital and community, suboptimal infection prevention and control practices, use of invasive devices, prolonged hospital stay and a myriad of immunosuppressive conditions are common in our setting [6]. This study was therefore carried out to determine the distribution of Klebsiella pneumoniae carbapenemases among clinical isolates of Klebsiella pneumoniae in UPTH.

\section{Methods}

\subsection{Study Design}

This was a cross-sectional study conducted in the
Department of Medical Microbiology and Parasitology, University of Port Harcourt Teaching Hospital. Rivers state, Nigeria, between December 2017 and April 2018.

\subsection{Sampling and Specimen Collection}

Klebsiella pneumoniae isolates from patients on hospital admission were recruited consecutively till the sample size of 225 was achieved. The minimum sample size of 225 (using a prevalence of $16.7 \%$ ) [12] was derived based on the calculation for cross-sectional descriptive studies [14]. Source samples include urine, wound swabs, body fluid aspirates, blood and Cerebrospinal fluid from patients admitted into pediatric and adult medical and surgical wards as well as the Intensive care units.

\subsection{Data Collection}

Basic demographic information were obtained from the laboratory forms and include Age, Sex, Ward/clinic and Provisional diagnosis. Relevant information not contained in the laboratory form was obtained from patients' folders or patients/care-givers.

\subsection{Specimen Analyses}

Specimens including urine, blood, Cerebrospinal fluid, wound and body fluid aspirates submitted from in-patients to the Medical Microbiology and Parasitology laboratory for microscopy, culture and sensitivity testing were inoculated unto MacConkey agar plates and incubated aerobically at 35$37^{\circ} \mathrm{C}$ for $16-18$ hours. Pure colonies of isolates were subjected to Gram staining, motility and biochemical tests. Lactosefermenting Gram negative, non-motile, citrate positive and indole negative bacilli were presumptively identified as Klebsiella pneumoniae. These were confirmed using MICROBACT 12A identification kits (Oxoid, UK), according to the manufacturer's instructions. A total of six hundred and fifty (650) clinical specimens were analyzed from which 225 Klebsiella pneumoniae isolates required for the study were derived. Only one isolate was recruited from each patient.

Antimicrobial susceptibility testing to the carbapenems using Ertapenem $(10 \mu \mathrm{g}$ disc, Oxiod England) as a surrogate, was performed on all isolates of Klebsiella pneumoniae using the modified Kirby Bauer disk diffusion technique according to Clinical Laboratory Standards Institute guidelines [15]. Zones of inhibition were measured and interpreted as resistant, intermediate or susceptible using criteria recommended by CLSI [16]. All Klebsiella pneumoniae isolates which were found to be intermediate or resistant to Ertapenem were considered to be suspected carbapenemase producers and these were subjected to phenotypic confirmatory testing by the

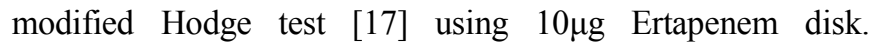
Klebsiella pneumoniae ATCC BAA-1706 and Klebsiella pneumoniae ATCC BAA-1705 were used as negative and positive control respectively for all diagnostic processes.

\subsection{Molecular Studies}

Following extraction and quantification, KPC genes from 
the isolates were amplified using the KPC forward (5'-GCT CAG GCG CAA CTG TAA G-3') and reverse primers (5'AGC ACA GCG GCA GCA AGA AAG-3') on an ABI Thermal cycler 9700 (Applied Biosystems) at a final volume of $40 \mu \mathrm{l}$ comprising $20 \mu 1$ master mix, $0.64 \mu \mathrm{l}$ forward primer, $0.64 \mu \mathrm{l}$ reverse primer, $2 \mu \mathrm{l}$ template and $16.72 \mu \mathrm{l}$ nucleasefree water for 35 cycles. Each run contained negative control (nuclease-free water) and KPC-positive control (K. pneumoniae ATCC strain BAA-1705). The product was resolved on a $1 \%$ agarose gel at $130 \mathrm{~V}$ for 30 minutes and visualized on a UV transilluminator for a $150 \mathrm{bp}$ product size

PCR conditions included: Initial Denaturation $\left(95^{\circ} \mathrm{C}, 5\right.$ minutes); Denaturation $\left(95^{\circ} \mathrm{C}, 40\right.$ seconds); Annealing $\left(51^{\circ} \mathrm{C}, 40\right.$ seconds); Extension $\left(72^{\circ} \mathrm{C}, 40\right.$ seconds) and Final extension $\left(72^{\circ} \mathrm{C}, 5\right.$ minutes $)$.

\subsection{Data Analysis}

Data obtained was analyzed using the statistical package for social sciences (SPSS) version 25. The level of significance was set at 0.05 . Chi square test was used to estimate the possible association between blaKPC genes and the source sample and ward. Ethical Approval was obtained from the Ethical Committee of the University of Port Harcourt Teaching Hospital.

\section{Results}

Table 1 shows that $73.3 \%$ of isolates were from adults, $16 \%$ from children $(<18$ years) and $10.67 \%$ from neonates. $52.44 \%$ and $47.56 \%$ were from male and female patients respectively. The highest number of isolates were derived from urine samples and from patients on the surgical wards.

Table 1. Socio-demographic Distribution of Patients, Source Samples and Hospital Units.

\begin{tabular}{lll}
\hline Characteristic & Frequency $(\mathbf{n = 2 2 5})$ & Percentage $(\mathbf{\%})$ \\
\hline Age range & & \\
$\geq 18$ years & 165 & 73.33 \\
$<18$ years & 36 & 16.00 \\
$\leq 28$ days & 24 & 10.67 \\
Gender & & \\
Male & 118 & 52.44 \\
Female & 107 & 47.56 \\
Source Unit & & \\
SCBU & 25 & 11.1 \\
ICU & 29 & 12.89 \\
Paediatrics & 32 & 14.22 \\
Medicine & 61 & 27.11 \\
Surgery & 78 & 34.67 \\
Sample & & \\
Urine & 115 & 51.1 \\
Wound swab & 45 & 20 \\
Blood & 31 & 13 \\
Sputum & 21 & 9.3 \\
Body fluid aspirate & 10 & 4.4 \\
CSF & 5 & 1.3 \\
\hline
\end{tabular}

SCBU: Special care baby unit.

ICU: Intensive care unit
Figure 1 shows that $8.4 \%(19 / 225)$ isolates were not susceptible (intermediate or resistant) to Ertapenem, the surrogate used for the carbapenems.

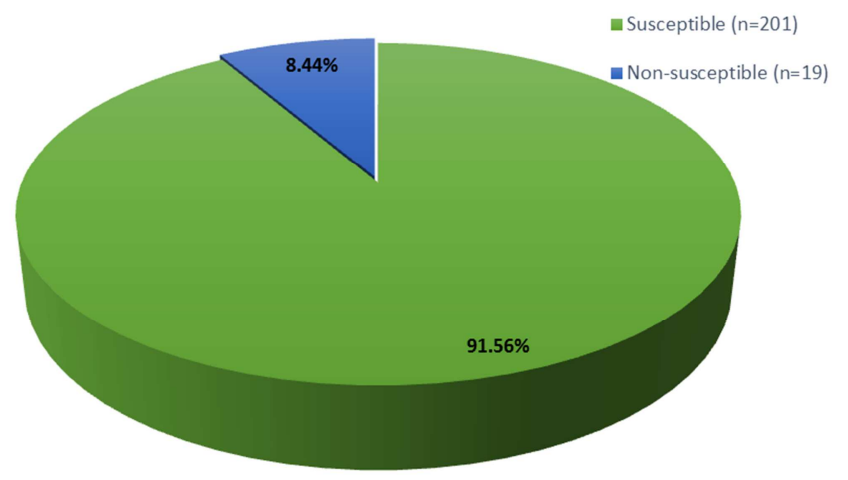

Figure 1. Susceptibility to Ertapenem.

Of these 19 non-susceptible isolates, 14 tested positive for carbapenemase production using the Modified Hodge Test, giving a phenotypic carbapenemase prevalence of $6.2 \%$ (Table 2).

It is interesting to note here that all the MHT positive isolates were out rightly resistant to Ertapenem (Table 2). Those isolates that were intermediately susceptible to Ertapenem, didn't express carbapenemase production phenotypically as shown by the MHT.

Table 2. Expression of Carbapenemases by non-susceptible K. pneumoniae isolates on MHT.

\begin{tabular}{llll}
\hline $\begin{array}{l}\text { Modified Hodge } \\
\text { Test (MHT) }\end{array}$ & Resistant & Intermediate & $\begin{array}{l}\text { Chi-square }(\boldsymbol{P} \text { - } \\
\text { value) }\end{array}$ \\
\hline Positive & $14(93.3)$ & $0(0.0)$ & \\
Negative & $1(6.7)$ & $4(100)$ & $14.18(0.00001)^{*}$ \\
Total & $15(100.0)$ & $4(100.0)$ & \\
\hline
\end{tabular}

*Distribution is statistically significant $(\mathrm{p}<0.05)$

Six (6) Klebsiella pneumoniae isolates only, expressed the blaKPC gene on PCR therefore the prevalence of blaKPC gene was $2.7 \%$ (Figure 2 ).

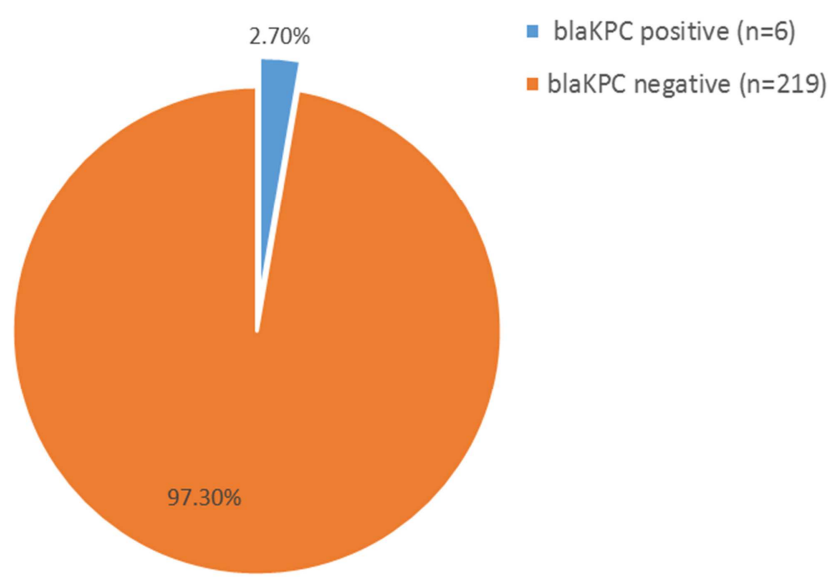

Figure 2. Prevalence of blaKPC genes among K. pneumoniae isolates.

Table 3 shows that more than half of the K. pneumoniae isolates that were not susceptible to Ertapenem by the 
conventional disc diffusion technique, did not express carbapenemase production as shown by both phenotypic and

genotypic confirmatory methods.

Table 3. Relationship between Phenotypic and Molecular findings.

\begin{tabular}{llll}
\hline blaKPC Gene & Number of isolates (\%) & MHT positive (\%) & Carbapenem non-susceptible (\%) \\
\hline Positive & $6(2.7)$ & $6(42.9)$ & $6(31.6)$ \\
Negative & $219(97.3)$ & $8(57.1)$ & $13(68.4)$ \\
Total & $225(100.0)$ & $14(100)$ & $19(100)$ \\
\hline
\end{tabular}

\section{Discussion}

The distribution of carbapenem resistant isolates according to source samples showed that $45.8 \%$ of the isolates were from urine, $29.2 \%$ from wounds and $12.5 \%$ from sputum. This is similar to the findings of Nagaraj et al. [18] who reported $42 \%$ of their KPC producing isolates were from urine, and $18 \%$ from wound infections. Schwaber et al. [19] also found that the most common clinical site of KPC producing Klebsiella pneumoniae infection is the urinary tract. This is probably due to frequent and prolonged urethral catheter usage as well as poor catheter insertion and management procedures in health care facilities.

The ICU and surgical wards were the highest contributors, accounting for $6.9 \%$ and $4 \%$ of the isolates respectively. This tallies with the report of Da Silva et al. [20] that the ICU has the highest percentage of carbapenemase producing Klebsiella pneumoniae isolates per ward in the health care facility. This is most likely related to the tendency of patients admitted into the ICU to have co-morbidities and to require a number of medical and surgical interventions including prolonged exposure to broad spectrum antibiotics which predispose them to infection by multi-drug resistant pathogens.

Resistance to the most commonly used antimicrobials has increased greatly among gram-negative bacteria, particularly of the Enterobacteriaceae family. Hitherto, ESBL production was the most worrisome form of resistance exhibited by this family [9]. Organisms with such gene(s) become multi-drug resistant hence limiting treatment options to only the Carbapenems [21]. It has however, now been shown that some ESBL genes also code for resistance to other classes of antibacterials.

Studies across the world have reported increased production of Klebsiella pneumoniae carbapenemases which hydrolyse all $\beta$-lactam antibiotics including the carbapenems [2]. This study revealed a $6.2 \%$ prevalence of carbapenemase phenotype and $2.7 \%$ prevalence of blaKPC among isolates of Klebsiella pneumoniae recruited. The low prevalence of KPC could be due to minimal usage of carbapenems in our setting as a result of their high cost and the non-availability of oral formulations which has protected them from being frequently subjected to abuse within and outside of the healthcare facility.

Among the non- susceptible isolates, only about one-third $(31.6 \%)$ were carbapenemase producing which suggests that other mechanisms of resistance (other than carbapenemase production) may be implicated. These other mechanisms of resistance to these antibiotics of last resort are not as much of a global public health concern because they are not plasmid mediated and as such transfer of these resistance genes within and between bacterial species is unlikely [22]. One of these mechanisms is porin loss which results in reduced permeability of the outer membrane and thereby hinders entry of the carbapenems into the cell [12]. This form of nonenzymatic resistance is frequent but its spread is limited because it is chromosomally mediated [23]. Resistance to Carbapenems could also be as a result of over expression of $\beta$-lactamases such as ESBLs and AmpC enzymes which express low-level carbapenemase activity [24]. This scenario is likely the case in this study since all the isolates that were of intermediate susceptibility to Ertapenem and MHT positive, were negative for the blaKPC gene.

Among the phenotypic carbapenemase producers found in this study using the MHT, only $42.9 \%(6 / 14)$ possessed the KPC gene, which implies that other carbapenemase genes which we did not test for, may have accounted for their carbapenemase phenotype. A similar study from Greece also reported that only $38 \%$ of their carbapenemase-producing Klebsiella pneumoniae isolates were found to be harboring KPC genes [7]. Our finding of $2.7 \%$ prevalence of blaKPC was higher than the zero prevalence reported by Mansoume et al. [25] Anderson et al [26] and Vasaikar et al. [27] as well as the $0.2 \%$ prevalence reported by Ehrhard et al. [28]. The low prevalence in these studies could be due to the fact that these were mainly surveillance studies conducted among asymptomatic persons. One wonders if it could also be due to restricted use of antibiotics in those countries as opposed to Nigeria where antibiotics are easily available over the counter, even without prescription. Alternatively, carbapenemase genes other than KPC could be the prevalent genes in their settings. It was however similar to the $2.9 \%$ prevalence reported by Parisi et. al. [29] in Italy but lower than the $3.8 \%$ prevalence reported in a similar study in Uganda [30], 31.1\% [31] in Egypt and 44.4\% [32] in Latin America. Nigerians tend to embark on more medical tourism trips to health care facilities in Asian countries due to lower travel and health care costs and this region has been reported to have a lower prevalence of KPC genes than Western countries where KPC enzymes are predominant [33].

As regards the disparity between the phenotypic and genotypic expression of carbapenemases by isolates in our study, Wang et al. reported that false positive results observed using the MHT probably occur due to low level hydrolysis of Ertapenem by ESBLs particularly those of the CTX-M type or high level AmpC $\beta$-lactamases [34]. These mechanisms probably contributed to some of the MHT 
positive, blaKPC negative isolates in our study. This is also similar to the findings of Anderson et al [26].

\section{Conclusion}

Critically ill patients are more likely to develop infections by blaKPC-producing Klebsiella pneumoniae. It is therefore pertinent that the approach to prevention and control of infections by multi-drug resistant Klebsiella pneumoniae isolates must be multi-pronged to effectively counteract the various mechanisms that may be responsible. From control of prescribing and dispensing patterns, rational use of antimicrobials, institution of antimicrobial stewardship programs, optimization of infection prevention and control measures to effective surveillance; a well-coordinated approach is necessary to reduce the prevalence and spread of these pathogens since the blaKPC resistance gene is plasmid mediated and associated with high rates of both inter and intra-species transfer among bacteria.

\section{References}

[1] Xu Z-Q. Flavin MT, Flavin J. Combating multidrug-resistant Gram negative bacterial infections. Expert Opin Investig Drugs. 2014; 23: 163-82.

[2] Nordmann P, Naas T, Poirel L. Global spread of Carbapenemase producing Enterobacteriaceae. Emerg Infect Dis. 2011; 17: 1791-1798.

[3] Poulou A, Voulgari E, Vrioni G, Koumaki V, Xidopoulos G, Chatzipantazi V, Markou F, et al. Outbreak Caused by an Ertapenem-Resistant, CTX-M-15-Producing Klebsiella pneumoniae ST101 Clone Carrying an OmpK36 Porin Variant. J. Clin. Microbiol. 2013; 51 (10): 3176-3182.

[4] Athanassios T, Ioulia K, Aggeliki P, Themeli-Digalaki K, Ikonomidis A, Petropoulou D et. Al. Evaluation of Boronic Acid Disk Tests for Differentiating KPC-Possessing Klebsiella pneumoniae isolates in the Clinical Laboratory. JCM. 2009; 362-367.

[5] Ramirez MS, Xie G, Marshall SH, Hujer KM, Chain PS, Bonomo RA et al. Multidrug-resistant (MDR) Klebsiella pneumoniae clinical isolates: a zone of high heterogeneity (HHZ) as a tool for epidemiological studies. Clin. Microbiol. Infect. 2012; 18: 254-258.

[6] Freire MP, Pierrotti LC, Filho HHC, Ibrahim KY, Magri AS, Bonazzi PR et al. infections with Klebsiella pneumoniae carbapenemase (KPC)-producing Klebsiella pneumoniae in cancer patients. Eur J Clin Microbiol infect Dis. 2015; 34: 277-86.

[7] Munoz-Price LS, Poirel L, Bonomo RA, Schwaber MJ, Daikos GL, Cormican M, et al. Clinical epidemiology of the global expansion of Klebsiella pneumoniae carbapenemases. Lancet Infect Dis. 2013; 13: 785-796.

[8] Paterson DL, Siu KL, Chang FY. Klebsiella species (K. pneumoniae, $K$. oxytoca, $K$. ozaenae and $K$. rhinoscleromatis); Antimcrobe. Available at http://www.antimicrobe.org/b107.asp. Accessed 5th September, 2019.
[9] Paczosa MK, Mecsas J. Klebsiella pneumoniae: going on the offense with a strong defense. Microbiol Mol Biol Rev 2016, 80 (3): 629-661.

[10] Manenzhe RI, Zar HJ, Nicol MP, Kaba M. The spread of carbapenemase-producing bacteria in Africa: a systematic review. J Antimicrob Chemother. 2015; 70 (1): 23-40.

[11] Onyedibe KI, Bode-Thomas F, Nwadike V, Afolaranmi T, Okolo MO, Uket $\mathrm{O}$ et al. High Rates of Bacteria Isolates of Neonatal sepsis with Multidrug Resistance patterns in Jos Nigeria. Ann pediatr Child Health. 2015; 3 (2): 1052.

[12] Hussaini IM, Olonitola OS, Suleiman AB. Occurrence of Carbapenem Resistant Klebsiella pneumoniae in Clinical Samples from Some Selected Hospitals in Zaria, Kaduna State, JAMPS. 2017; 13 (3): 1-11.

[13] Borer A, Saidel-Odes L, Riesenberg K, Eskira S, Peled N, Nativ R et al. Attributable mortality rate for carbapenemresistant Klebsiella pneumoniae bacteremia. Infect Control Hosp Epidemiol. 2009; 30: 972-6.

[14] Araoye MO. Sample size determination. Research methodology with statistics for health and social science. Ilorin, Nathadex publishers. 2004; 2: 115-121.

[15] Clinical and Laboratory Standards Institute. Performance Standards for Antimicrobial Susceptibility Testing. 27th ed. CLSI supplement M100-S28. Clinical and Laboratory Standards Institute, Wayne, PA. 2018.

[16] Performance standards for antimicrobial susceptibility Testing $26^{\text {th }}$ ed; informational supplement. M100S26 Clinical and Laboratory Standards Institute, Wayne, PA 2016.

[17] Cury AP, Andreazzi D, Maffucci M, Caiaffa-Junior HH, Rossi F. The modified Hodge test is a useful tool for ruling out Klebsiella pneumoniae carbapenemase. Clinics (Sao Paulo). 2012; 67 (12): 1427-1431. doi: 10.6061/clinics/2012(12)13.

[18] Nagaraj S, Chandran SP, Shamanna P, Macaden R. Carbapenem resistance among Escherichia coli and Klebsiella pneumoniae in a tertiary care hospital in south India. Indian $J$. Med. Microbiol. 2012; 30 (1): 93-95.

[19] Schwaber MJ, Klarfeld-Lidji S, Navon-Venezia S, Schwartz D, Leavitt A, Carmeli Y. Predictors of carbapenem-resistant Klebsiella pneumoniae acquisition among hospitalized adults and effect of acquisition on mortality. Antimicrob Agents Chemother. 2008; 52: 1028-1033.

[20] Da Silva KE, Maciel WG, Correia Sacchi FP, Carvalhaes CG, Rodrigues Costa F, da Silva ACR, Risk factors for KPCproducing Klebsiella pneumoniae: watch out for surgery. $J$ Med Microbiol. 2016; 65: 547-553.

[21] Girmenia C, Serrao A, Canichella M, Epidemiology of Carbapenem Resistant Klebsiella pneumoniae Infections in Mediterranean Countries. Mediterr J Hematol Infect Di. 2016; 8; e2016032.

[22] Gotoff SP. Sepsis in the newborn in Krugman, S, Katz SI, Gershon AA and Wilfert CM(ed.), Infectious diseases of children, $9^{\text {th }}$ ed. Mosby-Year Book, St. Louis, Mo. 1 992, 402-418.

[23] Padilla E, Llobet E, Domenech-Sanchez A, Martinez-Martinez L, Bengoechea JA, Alberti S. Klebsiella pneumoniae AcrAB efflux pump contributes to antimicrobial resistance and virulence. Antimicrob Agents Chemother. 2010; 54 (1): 177-183. 
[24] Potron A, Kalpoe J, Poirel L, Nordmann P. European dissemination of a single OXA-48-producing Klebsiella pneumoniae clone. Clin Microbiol Infect. 2011; 17: 24-6.

[25] Masoume B, Abazar P, Shiva M, Malihe T, Gholamreza I. Detection of the Klebsiella pneumoniae carbapenemase in $K$. pneumoniae isolated from the Clinical Samples by the Phenotypic and Genotypic Methods. Iran J Pathol. 2015; 10 (3): 199-205.

[26] Anderson KF, Lonswy DR, Rasheed JK, Biddle J, Jensen B, McDougal LK, et al.; Evaluation of method to identify the Klebsiella pneumoniae carbapenemase in enterobacteriaceae. J. Chin microbial. 2007; 45: 2723-2725.

[27] Vasaikar S, Obi L, Morobe I, Bisi-Johnson M. Molecular Characteristics and Antibiotic Resistance Profiles of Klebsiella Isolates in Mthatha, Eastern Cape Province, South Africa. Int J Microbiol. 2017; 8486742.

[28] Ehrhard L, Karaalp AK, Hackel T, Höll G, Rodewald N, Reif $\mathrm{U}$ et al. Prevalence of carbapenemase-producing bacteria in hospitals in Saxony, Germany. Bundesgesundheitsblatt Gesundheitsforschung Gesundheitsschutz 2014; 57 (4): 406413.

[29] Parisi SG, Bartolini A, Santacatterina E, Castellani E, Ghirardo R, Berto A, et al. Prevalence of Klebsiella pneumoniae strains producing carbapenemases and increase of resistance to colistin in an Italian teaching hospital from
January 2012 To December 2014. BMC Infect Dis. 2015; 15: 244; PMID: 26116560.

[30] Okoche D, Asiimwe BB, Katabazi FA, Kato L, Najjuka CF. Prevalence and Characterization of Carbapenem-Resistant Enterobacteriaceae Isolated from Mulago National Referral Hospital, Uganda. PLoS ONE. 2015; 10 (8): e0135745. doi: 10.1371/journal.pone.0135745

[31] Metwally L. Gomaa N, Attallah M, Kamel N. High prevalence of Klebsiella pneumoniae carbapenemase-mediated resistance in K. pneumoniae isolates from Egypt. EMHJ. 2013; 19 (11): 947-952.

[32] Villegas MV, Pallares CJ, Escandon-Vargas K, HernandezGomez C, Correa A, Alvarez C et al. Characterization and clinical impact of bloodstream infection caused by carbapenemase- producing enterobacteriaceae in seven latin American countries. PLoS One. 2016; 11: e0154092; PMID: 27104910 .

[33] Naas T, Cuzon G, Villegas MV, Lartigue MF, Quinn JP, Nordmann P. Genetic structures at the origin of acquisition of the b-lactamase blaKPC gene. Antimicrob Agents Chemother. 2014; 52: 1257-1263.

[34] Wang P, Hu F, Xiong Z, Ye X, Zhu D, Wang YF, et al, Susceptibility of extended spectrum $\beta$-lactamase producing Enterobacteriaceae according to the new CLSI breakpoints. $J$ Clin Microbiol. 2011; 49: 3127-3131. 